\title{
PhenX: Host: Social/Cognitive measures for tobacco regulatory research
}

\author{
Megan E Piper, ${ }^{1}$ Darigg C Brown, ${ }^{2}$ Tabitha P Hendershot, ${ }^{2}$ Gary E Swan, ${ }^{3}$ PhenX TRR \\ Host: Social/Cognitive Working Group
}

${ }^{1}$ Center for Tobacco Research and Intervention, University of Wisconsin-Madison, Madison, Wisconsin, USA

${ }^{2}$ RTI International, Research Triangle Park, North Carolina, USA

${ }^{3}$ Stanford Prevention Research Center, Stanford University School of Medicine, Stanford, California, USA

\section{Correspondence to}

Dr Megan E Piper, Center for Tobacco Research and Intervention, University of Wisconsin-Madison, Madison, WI 53711-2027, USA MEP@ctri.wisc.edu

Received 25 April 2018 Revised 29 June 2018 Accepted 2 July 2018
A) Check for updates

(c) Author(s) (or their employer(s)) 2020. No commercial re-use. See rights and permissions. Published by BMJ.

To cite: Piper ME, Brown DC, Hendershot TP, et al.

Tob Control 2020;29:s5-s12.

\section{ABSTRACT}

A working group (WG) of experts from diverse fields related to nicotine and tobacco addiction was convened to identify elements and measures from the Host: Social/ Cognitive domain to include in the Tobacco Regulatory Research Collection in the PhenX Toolkit, a catalogue of measures for biomedical research. This paper describes the methods used to identify, select, approve and include measures in the toolkit with potential relevance to users of both conventional and newer tobacco products, such as electronic cigarettes (e-cigarettes). In addition to 25 complementary measures primarily focused on cigarette use already present in the PhenX Toolkit, the WG recommended 11 additional social/cognitive measures focused on children and adult users or potential users of tobacco products. Of these, 10 were self-administered measures: frequency of communication with parents about smoking, quality of communication with parents about smoking, susceptibility to tobacco use, behaviour economics/purchase behaviour, motivation to quit (both single and multi-item measures), hedonic tone or response to pleasurable situations, multigroup ethnic identity, peer and family influence on smoking, attentional control and house rules about tobacco use. The remaining selected measure was computer based (distress tolerance). Although validated tools for use in the Host: Social/Cognitive realm are available, much remains to be done to develop, standardise and validate the tools for application to users of e-cigarettes and other non-combusted tobacco products, non-English language speakers and adolescents.

\section{INTRODUCTION}

Delnevo and Bauer ${ }^{1}$ reviewed the state-of-the-art of tobacco host-related surveillance and evaluation measures contained in 10 major national surveys, including the National Health Interview Survey, the Behavioral Risk Factor Surveillance System and the National Survey on Drug Use and Health. The inventory they presented $^{1}$ included 39 separate topic areas categorised into five domains (cigarette smoking behaviours, use of other tobacco products, youth issues, other and health behaviours/status/ consequences). No two surveys were consistent in terms of their measures in the five domains. Furthermore, those authors noted several gaps in the methodology used in the surveys, including a need for increased standardisation across measures, attention to non-English language surveys and surveys measuring the use of other tobacco products. They called for increased coordination and dissemination among researchers through a network in which host measures are catalogued and shared among public health investigators, such as tobacco monitoring and control. This paper represents an important step towards the establishment of a repository of recommended host measures.

The US Food and Drug Administration (FDA) and the Tobacco Regulatory Science Program of the National Institutes of Health (NIH) have partnered to expand the depth and breadth of tobaccorelated measures in the PhenX Toolkit. The PhenX Toolkit (https://www.phenxtoolkit.org/), a catalogue of recommended standard measures, can enhance cross-study analysis in large-scale research. To achieve this goal, a nine-member Tobacco Regulatory Research Panel (TRRP) was created to guide this endeavour. Working groups (WGs) were established to identify and select tobacco-related measures that could be included in the toolkit. Enhancing this repository with measures related to tobacco product use will allow scientists to use instruments to compare results across studies and combine data from distinct studies. This will allow for larger sample sizes, increased statistical power, and greater sample diversity and will contribute to furthering tobacco regulatory science. (See Swan et $a l$, this issue, for more details regarding this partnership and its goals and methodology.) This paper describes the development process of the PhenX TRR Host: Social/Cognitive WG and the resultant Specialty Collection of recommended consensus measures.

\section{METHODS \\ Determining scope of the TRR Host: Social/ Cognitive Collection}

The TRRP organised a series of WGs using the 'HAVE Model', an epidemiological framework that has been adapted for tobacco control surveillance. ${ }^{2}$ In this context, the terms 'Host', 'Agent', 'Vector' and 'Environment' derive from human epidemiological models of infectious disease where the Hosts (children and adults) are affected by disease-causing Agents (tobacco products) transmitted by the Vector (tobacco industry and retailers) within the context of the Environment. This WG was charged with identifying the intrapersonal factors that influence product use, including cognitive, affective, motivational and perceptive factors (ie, perceived norms and risk perceptions), from the list generated by the TRRP. In other words, this WG was asked to review several potential elements that, in its best judgement, could characterise the 'host' or person who does, or would, use tobacco products (table 1).

The initial scope outlined in table 1 was generated as a 'working draft' by the overarching TRRP 
Table 1 Initial Host: Social/Cognitive scope

\begin{tabular}{ll}
\hline Acculturation status/cultural identity & Motivation to quit \\
\hline Attention & Motives/reasons for use \\
\hline Attitudes & Nicotine dependence/tolerance \\
\hline Behaviour economics/purchase behaviour & Parental monitoring \\
\hline Cessation self-efficacy & Perceived norms \\
\hline Current mood state & Perceived prevalence \\
\hline Decision-making/delayed discounting & Peer influence/smoking \\
\hline Expectancies & Personality \\
\hline Health literacy & Reasons to switch products \\
\hline $\begin{array}{l}\text { Information seeking behaviour (web, } \\
\text { smartphone) }\end{array}$ & Risk perceptions \\
\hline Intentions to use/susceptibility & Stress \\
\hline Knowledge & Subjective effects of products \\
\hline Memory & \\
\hline
\end{tabular}

to start the consensus process engaged in by the WG. The WG recognised that measures of some of the elements were included in the toolkit by previous, non-tobacco regulatory WGs (see table 2), while other measures were subsumed by other tobacco regulatory WGs. Discussion then focused on the selection of

Table 2 Complementary TRR Host: Social/Cognitive measures already in the PhenX Toolkit

\begin{tabular}{|c|c|}
\hline PhenX measure (PhenX ID) & Domain/Collection name \\
\hline Tobacco (non-cigarette)-Product Use (081401) & Oral Health (Domain) \\
\hline $\begin{array}{l}\text { Protocol 1: Adolescent Tobacco Use } \\
\text { 30-Day Quantity and Frequency (030803) } \\
\text { Age of Initiation Use (030701) } \\
\text { Age of Offset of Use (030903) } \\
\text { Smoking Status (030603) }\end{array}$ & \multirow[t]{3}{*}{$\begin{array}{l}\text { Alcohol, Tobacco and Other } \\
\text { Substances (Domain) }\end{array}$} \\
\hline $\begin{array}{l}\text { Protocol 2: Adult Tobacco Use } \\
30 \text {-Day Quantity and Frequency (030804) } \\
\text { Age of Initiation Use (030703) } \\
\text { Age of Offset of Use (030902) } \\
\text { Smoking Status (030604) } \\
\text { Cigarette Nicotine Dependence (031001) }\end{array}$ & \\
\hline $\begin{array}{l}\text { Substances } \\
\text { Lifetime Abuse and Dependence (031401) } \\
\text { Lifetime Use (031101) }\end{array}$ & \\
\hline Urine Assay for Tobacco Smoke Exposure (091701) & Respiratory (Domain) \\
\hline $\begin{array}{l}\text { Smoking Quit Attempts (071001) } \\
\text { Personal Perception and Knowledge of Smoking- } \\
\text { Related Cancer Risk (070701) }\end{array}$ & Cancer (Domain) \\
\hline $\begin{array}{l}\text { Patterns of Substance Use } \\
\text { Adolescents (510303) } \\
\text { Adults (510302) }\end{array}$ & \multirow[t]{2}{*}{$\begin{array}{l}\text { Assessment of Substance Use } \\
\text { and Substance Use Disorders } \\
\text { (Specialty Collection) }\end{array}$} \\
\hline $\begin{array}{l}\text { Substance Abuse and Dependence-Past Year- } \\
\text { Tobacco (510403) }\end{array}$ & \\
\hline $\begin{array}{l}\text { Expectancies-Alcohol, Tobacco, and Other } \\
\text { Substances-Tobacco }(520407) \\
\text { Motives-Alcohol, Tobacco, and Other Substances- } \\
\text { Specific-Tobacco ( } 520504)\end{array}$ & $\begin{array}{l}\text { Substance-Specific } \\
\text { Intermediate Phenotypes } \\
\text { (Specialty Collection) }\end{array}$ \\
\hline Disapproval of Substance Use (550202) & $\begin{array}{l}\text { Substance Use-Related } \\
\text { Community Factors (Specialty } \\
\text { Collection) }\end{array}$ \\
\hline $\begin{array}{l}\text { Current Environmental Tobacco Smoke Exposure } \\
\text { (060701) }\end{array}$ & $\begin{array}{l}\text { Environmental Exposures } \\
\text { (Domain) }\end{array}$ \\
\hline Personality Traits (121101) & Psychiatric (Domain) \\
\hline $\begin{array}{l}\text { Disinhibiting Behaviors-Impulsivity (Child, 180402; } \\
\text { Adolescent and Adult, 180401) }\end{array}$ & Psychosocial (Domain) \\
\hline
\end{tabular}

measures with supportive psychometric data, broad applicability, reasonable respondent burden, public availability and logistical feasibility. Following this discussion, the final list of measures (see table 3) was necessarily smaller in scope.

\section{Expertise of the Host: Social/Cognitive WG}

The Host: Social/Cognitive WG consisted of five experts with established research portfolios and expertise in the psychosocial risk factors for tobacco use and their impact on the tobacco regulatory environment. Two of the members served as co-chairs of the WG to lead the consensus process and provide guidance for the selection of standardised measures. The WG was supported by two liaisons from the TRRP, one special consultant and two federal agency (FDA and NIH) experts who provided input regarding the federal government's perspective on tobacco regulatory priorities and how they meshed with current research on the social and cognitive measures used to examine tobacco use and inform prevention strategies.

The expertise of the WG members spanned several areas of inquiry, including racial, ethnic and cultural risk factors related to tobacco use; health disparities; trajectories of tobacco use; resilience and dependence; smoking cessation; addictive behaviours; risk perceptions and attitudes; and health messaging. In addition, several of the WG members had experience with special populations (eg, adolescents, young adults and marginalised populations). The expertise of the WG involving these special populations was used during the consensus process to prioritise and select measures for inclusion in the toolkit. This expertise also helped ensure adequate coverage of the most pressing areas for which standard measures and protocols to assess the social and cognitive elements of tobacco users were lacking in the current PhenX Toolkit. The breadth and depth of the expertise of the WG helped fulfil the aim of PhenX to expand the scope of the toolkit by establishing recommended measures in support of tobacco regulatory research (TRR) and, thereby, accelerating the integration of social/cognitive measures into new and ongoing TRR studies.

\section{WG deliberations}

The specific goal for the Host: Social/Cognitive WG was to identify validated measures that assessed the scope discussed below for inclusion in the PhenX Toolkit. The primary selection criteria were that the measures be (1) clearly defined, (2) well established, (3) broadly applicable and generally acceptable, (4) low burden to participants and investigators, (5) broadly validated with demonstrated utility, (6) reproducible, (7) specific, (8) reliable and (9) have a standard measurement protocol. The WG also considered other criteria, including the following: (1) cross-cutting relevance for population groups, (2) relevance to TRR, (3) use in major reference studies (eg, the National Health and Nutrition Examination Survey), (4) open-source software and non-proprietary instruments (preferred), (5) brevity and (6) expectation of acceptance by the research community.

During their introductory call on 7 March 2015, the WG started the process of selecting measures by reviewing what was already in the toolkit. This review provided insight into where the WG should focus their efforts to identify new measures that were not already addressed by the toolkit. The measures listed in table 2 were already in the toolkit when the WG was formed and were complementary to the group's scope. PhenX identification numbers are provided in the table to facilitate reader access to the location of each measure in the toolkit. 
Table 3 The 11 TRR Host: Social/Cognitive measures recommended and approved for inclusion in the PhenX Toolkit

\begin{tabular}{|c|c|}
\hline Measure name (PhenX ID) & Description of measurement protocol \\
\hline Quality of Communication about Smoking (710101) & $\begin{array}{l}\text { This self-administered protocol asks an adolescent (12-17years) about the quality of communication with his/her } \\
\text { parent about smoking. }\end{array}$ \\
\hline Frequency of Communication about Smoking (710201) & $\begin{array}{l}\text { This self-administered protocol asks an adolescent (12-17 years) about the frequency of communication with his/ } \\
\text { her parent about smoking. }\end{array}$ \\
\hline Susceptibility to Smoking Cigarettes (710301) & $\begin{array}{l}\text { This self-administered protocol assesses the likelihood that adolescents or young adults (12-22 years) who have } \\
\text { never smoked will start smoking. }\end{array}$ \\
\hline Behaviour Economics/Purchase Behaviour (710401) & $\begin{array}{l}\text { This self-administered simulation protocol (14years and older) assesses the reinforcement efficacy of cigarettes } \\
\text { within a specific context. }\end{array}$ \\
\hline Motivation to Quit-single and multi-item & $\begin{array}{l}\text { The self-administered single-item protocol (14years and older) assesses the motivation to quit smoking among } \\
\text { those who want to quit smoking (710501). The self-administered multi-item protocol (14years and older) } \\
\text { assesses specific motivations for smoking cessation ( } 710502) \text {. }\end{array}$ \\
\hline Hedonic Tone or Response to Pleasurable Situations (710601) & $\begin{array}{l}\text { This self-administered protocol (18years and older) assesses four domains of the pleasure response/hedonic } \\
\text { experience. }\end{array}$ \\
\hline Distress Tolerance (710701) & $\begin{array}{l}\text { This computer-assisted protocol (18years and older) assesses distress tolerance as measured by the latency in } \\
\text { seconds to task termination. }\end{array}$ \\
\hline Multigroup Ethnic Identity (710801) & This protocol assesses multiethnic identity among adolescents and young adults (14-34 years). \\
\hline Peer and Family Influence on Smoking (710901)* & $\begin{array}{l}\text { This self-administered protocol assesses peer and family smoking among adolescents (12-17years) and young } \\
\text { adults (18-22 years). }\end{array}$ \\
\hline Attention (711001) & This self-administered protocol (18years and older) assesses attentional focusing and shifting. \\
\hline House Rules about Tobacco Use (711101) & $\begin{array}{l}\text { This interviewer-administered protocol ( } 12 \text { years and older) assesses whether, where and when tobacco use is } \\
\text { permitted inside the home. }\end{array}$ \\
\hline
\end{tabular}

*Selected for inclusion in the Core Tier 2 collection.

The WG members then selected and researched the scope described in table 1 and identified extant measures not already included in the PhenX Toolkit. The WG members presented their findings (ie, measures that assessed the targeted element) at a 2-day, in-person meeting where various measures were reviewed and discussed using the selection criteria described above. The WG then conducted additional literature review and further examination of the extant PhenX measures. The findings were then discussed during two teleconferences and via numerous email exchanges. At the conclusion of this process, the WG came to a consensus that the following 11 measures should be considered for inclusion in the toolkit: (1) behaviour economics/purchase behaviour, (2) quality of communication about smoking, (3) attention, (4) peer and family influence on smoking, (5) hedonic tone or response to pleasurable situations, (6) susceptibility to smoking cigarettes, (7) multigroup ethnic identity, (8) frequency of communication about smoking, (9) motivation to quit-single and multi-item, (10) distress tolerance and (11) house rules about tobacco use.

A number of the elements in the Host: Social/Cognitive domain are multidimensional in nature and some overlap with other domains was unavoidable. For example, a child's cognitive processing of parental monitoring (eg, permissive or restrictive) will influence the child's smoking behaviour. Similarly, nicotine dependence is widely seen as having multiple components including those that are contextual and cognitive in nature. Thus, nicotine dependence, as an element, could occur within the scope of more than one WG.

The primary authors of the measurement protocols suggested for inclusion were contacted to seek permission for their potential inclusion in the PhenX Toolkit. Additionally, the proposed measures were shared with the research community as part of the Community Outreach, in which an email was sent to researchers in academia and government agencies and those affiliated with various professional societies and scientific journals to obtain their comments about the proposed PhenX Toolkit measures. This feedback aided in refining the selection of measures for the toolkit and building consensus and acceptance of the measures throughout the research community. Additionally, this outreach facilitated obtaining opinions regarding the priority and appropriateness of the proposed measures and the utility of the measurement protocols. All feedback was reviewed and considered, although the WG did not agree with all comments and subsequently not all feedback was incorporated. The feedback was used by the WG to make their final selection of measures to include in the PhenX Toolkit, which occurred on 9 July 2015. (See Swan et al, this issue, for additional details about this process.)

\section{RESULTS}

\section{Community Outreach}

Seventeen people responded to the Community Outreach email and 12 provided ratings on the value of the various measures recommended. Overall, the respondents rated the proposed measures as being valuable to TRR. However, some minor concerns were expressed about the following measures: multigroup ethnic identity (not consistent with Centers for Disease Control/Office of Management and BudgetDirective 15 on Race and Ethnicity), hedonic tone or response to pleasurable situations (may be tangential to tobacco regulation), frequency of communication about smoking (concerned about reliability), distress tolerance (measure may be too experimental) and attention (not relevant to tobacco regulation).

The WG discussed the feedback acquired via Community Outreach and decided on the final 11 measures to recommend for inclusion in the toolkit. These measures were subsequently approved by both the TRRP and the PhenX Steering Committee. The final 11 measures, which were the same 11 sent for Community Outreach, were released into the toolkit on 20 February 2015. Table 3 gives a brief description of each measure. The toolkit provides the measure, the populations and tobacco products it has been validated for use with and scoring procedures (if applicable) (http:/www.phenxtoolkit.org). PhenX identification numbers are provided at the beginning of each section below to facilitate reader access to each recommended measure's location within the PhenX Toolkit. 


\section{Final Host: Social/Cognitive measures included in the PhenX Toolkit}

Quality of Communication about Smoking (PhenX ID=710101).

This is a series of six self-administered questions geared towards asking an adolescent (12-17 years) about the quality of communication with his/her parent about smoking (ie, mutual interest in each other's opinions, ease of discussion, comfort during discussion, perception of parent's honesty and reasonableness, feeling of being understood and feeling of being taken seriously). The response options are on a 5-point Likert scale ranging from $1=$ completely not true to $5=$ completely true. The questions have shown predictive validity in numerous studies, are low burden and are freely available. ${ }^{3}$

Frequency of Communication about Smoking (PhenX $\mathrm{ID}=710201)$. This is a series of eight self-administered questions that ask an adolescent (12-17 years) about communication with his/her parent about smoking. ${ }^{3}$ The response options are on a 5 -point Likert scale ranging from $0=$ never to $4=$ very often. Items assess communication about refraining from smoking, discouragement of friends who smoke, the dangers of smoking, possible actions concerning tobacco use, potential punishment for smoking, specific instructions to not smoke, the portrayal of smoking in movies and the importance of resisting peer pressure to smoke. The questions have shown predictive validity in numerous studies, are low burden and are freely available. ${ }^{45}$

Susceptibility to Smoking Cigarettes (PhenX ID=710301). This 3-item instrument is used to predict whether adolescents or young adults (12-22 years) who have never smoked are likely to start smoking. Item responses are on a 4-point scale-'definitely yes', 'probably yes', 'probably not' and 'definitely not'-and include 'refused' and 'don't know' options. ${ }^{6}$ A gateway question-'Smoking Status', an existing PhenX Toolkit measuremust be asked first. This protocol should only be used if the respondent has never smoked. Participants who are currently non-smokers and answer 'definitely not' to all four items are classified as non-susceptible; all remaining participants are considered susceptible to smoking. The protocol can be used across gender and race/ethnicity and requires no training or equipment to administer. This scale has also been used to predict smoking among young adults, including military recruits.

Behaviour Economics/Purchase Behaviour (PhenX $\mathrm{ID}=710401)$. The Cigarette Purchase Task $(C P T)^{7}$ is a selfadministered simulation procedure to assess the reinforcement value of cigarettes within a specific context in respondents 14 years and older. It is a time-efficient and cost-efficient assessment of the relative reinforcing efficacy of cigarettes that has good reliability and test-retest stability and is related to dependence and cigarettes per day. ${ }^{89}$ The CPT came from an adaptation of a progressive-ratio operant schedule.

Current smoking status must be ascertained before implementing this protocol, which is intended for use with current smokers. The CPT consists of both state (right now) and trait (a typical day) assessments of how many cigarettes the respondent would consume if they cost various amounts of money (ranging from US $\$ 0 /$ pack to US $\$ 22400 /$ pack) and under a variety of assumptions. For adolescents, alternate prices can be considered from those used for adults. ${ }^{9}$ The data result in five demand indices.

Motivation to Quit (single (PhenX ID=710501) and multiitem $(P h e n X I D=710502)$ ). The single-item version asks the respondent to indicate on a 10-point scale how motivated she/ he is to quit smoking ( $1=$ not at all motivated to $10=$ extremely motivated). This item is only intended for use in current smokers and has been shown to be related to quit attempts in adults and quit attempts and cessation among adolescents (14 years and older) as an important indicator of validity. ${ }^{10} 11$ This item is also frequently used to screen smokers for smoking cessation trials.

For a multi-item motivation assessment, the WG recommended the Reasons for Quitting (RFQ) ${ }^{12}$ Questionnaire, which is a 20-item self-administered protocol developed to assess specific motivations for smoking cessation. The respondent is asked to rate the extent to which each motivation describes them $(0=$ not at all true and $4=$ extremely true). The RFQ is based on an intrinsic-extrinsic model of motivation for smoking cessation with 10 intrinsic items (eg, health concerns and selfcontrol) and 10 extrinsic items (eg, immediate reinforcement and social pressure). The RFQ has good psychometrics, is multidimensional and assesses motivations for quitting, such as short-term consequences, social disapproval and long-term consequences. An adolescent (aged 14-19 years) version has also been developed. ${ }^{13}$

This protocol should be used only if the subject is a current or former smoker. This questionnaire is most appropriate for tobacco users who are in some way motivated to quit smoking. With appropriate changes in the introductory wording, researchers could also administer this questionnaire to smokers who have either previously quit or are currently smoking but are not interested in quitting. ${ }^{14}$

Hedonic Tone or Response to Pleasurable Situations (PhenX $\mathrm{ID}=710601$ ). Anhedonia, the inability to experience pleasure in normally pleasurable acts, is associated with smoking cessation outcomes. ${ }^{15}{ }^{16}$ Emotional regulation, and specifically anhedonia, may be a core dimension of depression associated with cessation failure. ${ }^{15}{ }^{17}$ The WG selected the Snaith-Hamilton Pleasure Scale (SHAPS), ${ }^{18}$ a self-administered, paper-and-pencil questionnaire with 14 items assessing four domains of pleasure response/ hedonic experience: interest/pastimes, social interaction, sensory experience and food/drink in adults (18 years and older). The WG acknowledges that this protocol was developed in English as used in the UK and recommends adjusting for English as used in the USA as needed.

In this protocol, the respondent is asked to indicate the extent to which he/she agrees ('agree' or 'strongly agree') or disagrees ('disagree' or 'strongly disagree') to each of 14 potentially pleasurable situations. A higher total SHAPS score indicates a higher level of anhedonia.

Distress Tolerance (PhenX ID=710701). Distress tolerance is the ability to persist in a goal-directed activity when experiencing psychological distress or the ability to tolerate psychological distress in general. One measurement approach is time spent on a frustrating behavioural task (ie, task persistence). Distress tolerance appears to be a relatively stable trait. Across measures, it has been found to be predictive of substance-use outcomes, including smoking cessation. ${ }^{19}{ }^{20}$ Individuals who have low distress tolerance may experience greater difficulty in maintaining tobacco abstinence in the face of withdrawal and other challenges. ${ }^{21}$

The Mirror Tracing Persistence Task (MTPT) has been demonstrated to discriminate between adult smokers and non-smokers and predict smoking cessation outcomes. ${ }^{19} 2223$ The original MTPT $^{23}$ required the construction or purchase of a physical box with a slanted mirror on one side. The participant inserts his or her hand and traces geometric figures while only watching the hand in the mirror. This is extremely difficult and frustrating. Persistence is scored as the mean time spent on several trials prior to giving up. The predictive validity of task persistence as assessed by the MTPT has been published in the context of 
smoking cessation outcomes among adults ${ }^{19} 22$ but not among adolescents.

The Computerised MTPT (MTPT-C) ${ }^{24}$ was developed to make the test more accessible; indeed, the MTPT-C does not require tracking equipment to conduct the assessment. Thus, this test has potential as a screening instrument to identify individuals at high risk of smoking initiation or smoking cessation failure. To date, the MTPT-C has been used to predict treatment dropout among substance abusers and shown to be correlated with other distress tolerance tasks among smokers. ${ }^{25}$ Distress tolerance is measured by the latency in seconds to task termination. Several quantitative indices of tracing accuracy, duration and time to completion in each of the modes are provided.

Multigroup Ethnic Identity (PhenX ID=710801). The WG selected a general measure to assess both cognitive and affective components of a respondent's sense of belonging and connectedness to a group of people with a common history, a clear understanding of the meaning of one's group membership, positive attitudes towards the group, familiarity with its history and culture, and involvement in its practices. ${ }^{26}$ Ethnic identity development begins in childhood, and by young adulthood, identities have been reached; that is, people have a secure sense of themselves as members of a group. However, some evidence suggests that further development occurs during adulthood. ${ }^{2728}$

The Ethnic Identity Scale is a 12-item multigroup ethnic identity measure used among adolescents and young adults (aged 14-34 years) to assess positive ethnic attitudes and sense of belonging ( 5 items); ethnic identity achievement, including exploration and resolution of identity issues (7 items) and ethnic behaviours and practices ( 2 items). In recent years, Phinney proposed the Multigroup Ethnic Identity Measure-Revised, which is a shorter, 10 -item version ${ }^{29}$; however, it has not been well validated.

Peer and Family Influence on Smoking (PhenX ID=710901). The measure selected for this element assesses adolescent (12-17 years) and young adult (18-22 years) evaluation of the use of cigarettes by parents and siblings and perception (not actual use) of the number of friends who use tobacco in their best-friend social network. This measure focuses on the exposure of the respondent to smoking in their immediate social networks and can be either a self-administered or intervieweradministered questionnaire. The 5-item National Cancer Institute (NCI) measure consists of five questions about peer and family smoking. ${ }^{30}$ Although it has not been used with people over the age of 22 years, the WG recommended that scientists consider using this measure with participants up to the age of 26 years, as the most recent Surgeon General's Report on young adult smokers, published in 2012, indicates that smoking initiation continues up to the age of 26 years. ${ }^{31}$

The WG recommends that these items be applied to other tobacco use (electronic cigarette (e-cigarette) and hookah), although the measure has only been validated in the context of cigarette use. Therefore, caution should be taken when applying it to other tobacco products.

Attention (PhenX ID=711001). Attention is the process of selecting specific aspects of the environment or ideas stored in memory for active cognitive processing. The WG selected the Attentional Control Scale (ACS), ${ }^{32} 33$ a 20-item self-report questionnaire developed to measure general individual differences in attentional focusing and shifting in adults 18 years and older who are known to be influenced by nicotine and other substances found in tobacco products. The ACS is brief and easy to administer and has solid psychometric properties and supportive validity information.
House Rules about Tobacco Use (PhenX ID=711101). This protocol includes questions from the Wave 1 Adult Population Assessment of Tobacco and Health (PATH) Study instrument (PATH Study Wave 1 Adult Extended Interview 7.5. Items AR1045 and AR1050). ${ }^{34}$ Two interviewer-administered questions ask whether or not smoking combustible (ie, cigarettes, cigars, pipes or hookah) and non-combustible tobacco products (ie, smokeless tobacco, dissolvable tobacco and e-cigarettes) is allowed inside the home ('at no time and in no place', 'some places or times' or 'anywhere and anytime'). These two questions can be asked of both adult and adolescent populations (12 years and above).

\section{DISCUSSION}

The goal of the Host: Social/Cognitive WG was to identify validated assessments that target key social and cognitive factors, were not already included in the PhenX Toolkit and are relevant for TRR. Key tobacco-specific measures, such as use patterns, dependence, motives, expectancies and exposure, and more general person-factor measures, such as the Big 5 Personality assessment and measures of impulsivity/sensation seeking, were already covered in the toolkit. Thus, the Host: Social/Cognitive WG could expand the toolkit by selecting novel tobacco-specific and general social/cognitive measures related to tobacco use and regulatory research.

The WG identified protocols that target a variety of tobaccospecific host constructs that influence initiation, regular use, dependence and the ability to quit using tobacco products. For instance, household family and peer influence on smoking (Quality of Communication about Smoking, Frequency of Communication about Smoking, Peer and Family Influence on Smoking and House Rules about Tobacco Use) and likelihood of initiating use (Susceptibility to Smoking Cigarettes) all target individual difference variables that could influence the initiation and continued use of a tobacco product. Such data could be important for research on the development of use patterns of different tobacco products. Motivation to quit (single and multi-item) and the willingness to give up monetary reward to use a product (ie, the rewarding value of a tobacco product; Behaviour Economics/Purchase Behaviour) could provide insight into the degree of dependence on a product and the likelihood that someone would try to quit using a product and ultimately be successful; these are critical questions when evaluating tobacco products.

The WG also identified host measures that are independent of tobacco products but can provide reliable and valid characterisations of the sample being studied. These measures were chosen to complement the existing measures in the PhenX Toolkit, such as the Big 5 Personality assessment and measures of impulsivity/sensation seeking, and to provide insight into constructs that may be associated with tobacco product use. For instance, the WG included measures that assess general affective constructs, such as response to rewards and positive life situations (hedonic tone or response to pleasurable situations), and the ability to tolerate discomfort, such as strong cravings or negative affect (distress tolerance). These measures are related to the initiation and maintenance of tobacco product use. The WG also selected a measure of attention focus and shifting that can be used to study the impact of tobacco use on attentional processing and as a risk factor for tobacco use. ${ }^{35}$ Finally, the WG included the Ethnic Identity Scale to provide a more comprehensive assessment of multiethnic identity among adolescents and young adults. 
In the process of selecting measures/protocols for inclusion in the Host: Social/Cognitive Speciality Collection, a variety of issues were discussed. One critical issue was the ability of extant, validated measures to assess constructs as they related to noncigarette tobacco products (eg, e-cigarettes, hookah and pipes). For instance, the theory behind the Behavioural Economics/ Purchase Behaviour task is relevant to all tobacco products. ${ }^{36} 37$ However, the quantity of tobacco used varies by product, and thus, the task must be modified slightly. Therefore, researchers must validate the measure for the different products (eg, how much would you pay for a cigarette vs a cartridge or puffing session with an e-cigarette vs minutes with a hookah). Another example of the need to specify assessments arose during the discussions of risk perceptions. Although this important topic can be assessed in a general sense, the validity of such measures is improved when they are focused on risks specific to the tobacco product in question. In the rapidly evolving market-place, the ability to reliably and validly assess measures relevant to novel product use will certainly be hindered by the speed with which scientists can develop and validate such measures. Therefore, the WG was careful to clarify which products can be reliably and validly assessed with the measures in the toolkit. However, it should be noted that ${ }^{1}$ very few measures have been validated with non-cigarette tobacco users.

Several measures were considered but not chosen to be included in the toolkit. The WG agreed that many of these measures are important to tobacco regulatory science. However, there was either insufficient literature regarding their psychometrics or validity or the assessments did not seem appropriate for the goals of the toolkit (ie, they were not brief assessments). For instance, the WG had hoped to include a measure of selfefficacy. A variety of self-efficacy measures were identified, many of which focused specifically on cessation self-efficacy or selfefficacy for resisting urges. ${ }^{38-43}$ However, a recent meta-analysis showed that a single item (eg, 'How confident are you that you will not be smoking in 1 year?') was often more predictive than multi-item measures. Unfortunately, the language of the single self-efficacy item used in most studies varied widely, and the WG was unable to find an item that was both clearly validated in the literature and predictive of cessation success. A request for assistance via Community Outreach did not identify any other potential measures.

Discussions also addressed methodological approaches that used excessive text or the use of percentages or other response options that rely on a numeracy that many people, especially adolescents, do not have. The issues of numeracy, general literacy and health literacy are significant concerns in assessment, ${ }^{44-46}$ especially for smokers who report less formal education. ${ }^{47}$ However, the WG was unable to identify brief, effective assessments of health literacy or numeracy.

Finally, the WG discussed how the Host: Social/Cognitive Collection would relate to the PATH Study and NCI's gridenabled measures (GEMs). The WG examined the Host-specific measures from the PATH Study to allow as much harmonisation with PATH Study data as possible. The WG also distinguished the GEM process, a bottom-up process driven by researchers attempting to coordinate their work, from the top-down PhenX process that enlists experts to identify measures and protocols that are already established in the field. Some attempt was made to examine how the PhenX Toolkit items and GEM complement each other. GEM is a wiki-based workspace platform that allows individuals to develop and publish multiple iterations of different measures. There is great potential to use GEM to provide a larger platform for tobacco regulatory measures. Moreover, GEM can serve as a repository for measures that are somewhat established or for expanding existing measures.

The WG identified a number of caveats in this work. First, many of the measures are applicable only to English-speaking populations. While the WG expects the underlying measures to be applicable beyond English speakers, more theoretical and psychometric work is needed to develop psychometrically based measures for use in non-English-speaking populations. While the intended consumers of the Host: Social/Cognitive measures included in the Toolkit are researchers working in the area of tobacco regulatory science, many of the validation studies used to select these measures were conducted in the USA. It is important to determine the psychometric properties and validity of these measures among non-English speakers and in non-Western cultures. Determination of the usability of various measures and their relevance to the tobacco users under study is critical and doing so is a 'best practice' for anyone conducting research in this area. Finally, the preferred method(s) of administration for each of the measures in the toolkit were identified and included based on available supportive psychometric evidence. Should a researcher choose to use a mode of administration other than those described in the toolkit for any measure, the reliability and other psychometric properties of that modality would need to be demonstrated.

In total, the Host: Social/Cognitive WG identified 11 validated measures that assess either tobacco specific or general 'Host' factors related to TRR. The consistent use of these measures in the PhenX Toolkit will facilitate tobacco science by encouraging researchers to use consistent, validated measures, and thereby obtain results that can be more easily compared across studies. This is especially important when researching novel tobacco products, for which multiple studies must be conducted and compared in a timely manner to provide a consistent research foundation on which regulatory decisions can be made.

\section{What this paper adds}

- Intrapersonal characteristics, such as cognitive, affective, motivational and perceptual characteristics (ie, perceived norms and risk perceptions), are well known to influence the initiation and maintenance of the use of tobacco products and thus are highly relevant to regulatory considerations.

- The continuous proliferation of novel tobacco products as advertised and marketed across a variety of platforms introduces challenges to scientists seeking to support evidence-based regulatory decision-making because suitable measures may not be readily available or widely known.

- The Host: Social/Cognitive Working Group filled previously noted gaps by providing, for the first time, consensus recommendations to investigators in the field, including best methods to assess the frequency and quality of communication with parents about smoking, susceptibility to tobacco use, purchase behaviour, motivation to quit, multigroup ethnic identity, peer and family influence on smoking and house rules about tobacco use.

- As tobacco regulatory science evolves, it will be critical for investigators to consistently use valid and comprehensive measures of multiple sources of Host: Social/Cognitive influence on tobacco product use.

Acknowledgements We gratefully acknowledge the contributions of the anonymous members of the scientific community who commented on the measures 
proposed for inclusion. We also acknowledge the contributions of Deborah Maiese, who led the recruitment of Working Group members and the orientation to the PhenX process, and the contributions of the RTI editorial team, including Debbie Bond, Loraine Monroe and Amy Morrow for providing expert editorial review of the manuscript.

Collaborators The following are members of the PhenX Tobacco Regulatory Research (TRR) Host: Social/Cognitive Working Group: Co-Chairs Thomas H Brandon, Moffitt Cancer Center, and Stephen T Tiffany, University at Buffalo; Working Group Members Laurie Chassin, Arizona State University, Pebbles Fagan, University of Arkansas for Medical Sciences and Megan Piper, University of Wisconsin-Madison; Special Consultant Bonnie Halpern-Felsher, Stanford University; TRR Panel Liaison Judith J Prochaska, Stanford University; TRR Panel Liaison and NIH Project Coordinator Kay L Wanke, NIH Office of Disease Prevention; NIH Liaison Annette Kaufman, National Cancer Institute; and the PhenX team from RTI International including Tabitha Hendershot, TRR RTI Project Lead, Darigg C Brown, WG Supervisor, and Lisa Kilpatrick, WG manager.

Contributors GES, TPH, and KLW led the conceptualisation of the manuscript and the coordination of the writing team. MEP and GES drafted the scientific content of the manuscript, and TPH and DCB drafted the Methods and Results sections of the manuscript. MEP, GES, TPH and KLW provided revisions. The Co-Chairs and Members of the PhenX TRR Host: Social/Cognitive Working Group identified and proposed preliminary measures, voted on final measures included in the PhenX TRR Host: Social/Cognitive Specialty Collection and reviewed the manuscript and provided substantive comments. TRR Panel members JJP and KLW ensured that the Working Group process maintained fidelity with overall project goals. Federal Agency Liaisons Sarah E Johnson, FDA Center for Tobacco Products and AK ensured project consistency with agency goals and priorities. NIH Project Coordinator KLW proposed the PhenX TRR initiative and contributed to its execution and completion. The PhenX team coordinated and facilitated the Working Group process, including project oversight and leadership (TPH), supervisory management (DCB) and project management (LK). Erin M Ramos, National Human Genome Research Institute, the PhenX NIH Program Official, provided project guidance and funding coordination. Carol M Hamilton, RTI International, is PhenX Principal Investigator and provided project guidance and supervision.

Funding Research reported in this publication was supported by grant numbers U41HG007050 and U41HG007050-01S1 from the NIH National Human Genome Research Institute and FDA Center for Tobacco Products.

Disclaimer The content is solely the responsibility of the authors and does not necessarily represent the official views of the $\mathrm{NIH}$ or the Food and Drug Administration

Competing interests None declared.

Provenance and peer review Not commissioned; externally peer reviewed.

\section{REFERENCES}

1 Delnevo CD, Bauer UE. Monitoring the tobacco use epidemic III: The host: data sources and methodological challenges. Prev Med 2009;48:516-23.

2 Giovino GA, Biener L, Hartman AM, et al. Monitoring the tobacco use epidemic I. Overview: optimizing measurement to facilitate change. Prev Med 2009;48:54-10.

3 Hiemstra M, Engels RC, Barker ED, et al. Smoking-specific parenting and smoking onset in adolescence: the role of genes from the dopaminergic system (DRD2, DRD4, DAT1 genotypes). PLoS One 2013;8:e61673.

4 Ennett ST, Bauman KE, Foshee VA, et al. Parent-child communication about adolescent tobacco and alcohol use: what do parents say and does it affect youth behavior? J Marriage Fam 2001;63:48-62.

5 Harakeh Z, Scholte RH, de Vries $\mathrm{H}$, et al. Parental rules and communication: their association with adolescent smoking. Addiction 2005;100:862-70.

6 Pierce JP, Choi WS, Gilpin EA, et al. Validation of susceptibility as a predictor of which adolescents take up smoking in the United States. Health Psychol 1996:15:355-61.

7 Jacobs EA, Bickel WK. Modeling drug consumption in the clinic using simulation procedures: demand for heroin and cigarettes in opioid-dependent outpatients. Exp Clin Psychopharmacol 1999;7:412-26.

8 Few LR, Acker J, Murphy C, et al. Temporal stability of a cigarette purchase task. Nicotine Tob Res 2012;14:761-5.

9 Murphy JG, MacKillop J, Tidey JW, et al. Validity of a demand curve measure of nicotine reinforcement with adolescent smokers. Drug Alcohol Depend 2011;113(23):207-14.

10 Turner $L R$, Mermelstein R. Motivation and reasons to quit: predictive validity among adolescent smokers. Am J Health Behav 2004;28:542-50.

11 Vangeli E, Stapleton J, Smit ES, et al. Predictors of attempts to stop smoking and their success in adult general population samples: a systematic review. Addiction 2011:106:2110-21.

12 Curry S, Wagner EH, Grothaus LC. Intrinsic and extrinsic motivation for smoking cessation. J Consult Clin Psychol 1990;58:310-6.
13 Myers MG, MacPherson L. Adolescent reasons for quitting smoking: initial psychometric evaluation. Psychol Addict Behav 2008;22:129-34.

14 Curry SJ, Grothaus L, McBride C. Reasons for quitting: intrinsic and extrinsic motivation for smoking cessation in a population-based sample of smokers. Addict Behav 1997;22:727-39

15 Leventhal AM, Piper ME, Japuntich SJ, et al. Anhedonia, depressed mood, and smoking cessation outcome. J Consult Clin Psychol 2014;82:122-9.

16 Dawkins L, Powell JH, Pickering A, et al. Patterns of change in withdrawal symptoms, desire to smoke, reward motivation and response inhibition across 3 months of smoking abstinence. Addiction 2009;104:850-8.

17 Leventhal AM, Zvolensky MJ. Anxiety, depression, and cigarette smoking: a transdiagnostic vulnerability framework to understanding emotion-smoking comorbidity. Psychol Bull 2015;141:176-212.

18 Snaith RP, Hamilton M, Morley S, et al. A scale for the assessment of hedonic tone the Snaith-Hamilton Pleasure Scale. Br J Psychiatry 1995;167:99-103.

19 Steinberg ML, Williams JM, Gandhi KK, et al. Task persistence predicts smoking cessation in smokers with and without schizophrenia. Psychol Addict Behav 2012:26:850-8.

20 Brown RA, Lejuez CW, Strong DR, et al. A prospective examination of distress tolerance and early smoking lapse in adult self-quitters. Nicotine Tob Res 2009;11:493-502.

21 Bernstein $\mathrm{A}$, Trafton J, Ilgen $\mathrm{M}$, et al. An evaluation of the role of smoking context on a biobehavioral index of distress tolerance. Addict Behav 2008:33:1409-15.

22 Brandon TH, Herzog TA, Juliano LM, et al. Pretreatment task persistence predicts smoking cessation outcome. J Abnorm Psychol 2003;112:448-56.

23 Quinn EP, Brandon TH, Copeland AL. Is task persistence related to smoking and substance abuse? The application of learned industriousness theory to addictive behaviors. Exp Clin Psychopharmacol 1996;4:186-90.

24 Strong DR, Lejuez CW, Daughters S, et al. The computerized mirror tracing task, version 1. Unpublished manual 2003.

25 Schloss HM, Haaga DA. Interrelating behavioral measures of distress tolerance with self-reported experiential avoidance. J Ration Emot Cogn Behav Ther 2011;29:53-63.

26 Phinney J. The multigroup ethnic identity measure: a new scale for use with diverse groups. J Adolesc Res 1992:7:156-76

27 Phinney JS. Ethnic identity exploration in emerging adulthood. In: Arnett JJ, Tanner JL, eds. Emerging adults in America: Coming of age in the 21st century. Washington, DC: American Psychological Association, 2006:117-34.

28 Ruble DN, Alvarez J, Bachman M, et al. The development of a sense of "we": the emergence and implications of children's collective identity. In: Bennett M, Sani F, eds. The development of the social self. New York, NY: Psychology Press, 2004:29-76.

29 Phinney JS, Ong AD. Conceptualization and measurement of ethnic identity: current status and future directions. J Couns Psychol 2007:54:271-81.

30 Pierce JP, Choi WS, Gilpin EA, et al. Tobacco industry promotion of cigarettes and adolescent smoking. JAMA 1998;279:511-5.

31 U.S. Department of Health and Human Services. Preventing tobacco use among youth and young adults: a report of the Surgeon General. Atlanta, GA: U.S. Department of Health and Human Services, Centers for Disease Control and Prevention, National Center for Chronic Disease Prevention and Health Promotion, Office on Smoking and Health, 2012

32 Derryberry D, Reed MA. Anxiety-related attentional biases and their regulation by attentional control. J Abnorm Psychol 2002;111:225-36.

33 Judah MR, Grant DM, Mills AC, et al. Factor structure and validation of the attentional control Scale. Cogn Emot 2014;28:433-51.

34 Hyland A, Ambrose BK, Conway KP, et al. Design and methods of the Population Assessment of Tobacco and Health (PATH) Study. Tob Control 2017;26:371-8.

35 Litten RZ, Ryan ML, Falk DE, et al. Heterogeneity of alcohol use disorder: understanding mechanisms to advance personalized treatment. Alcohol Clin Exp Res 2015;39:579-84

36 In: Bickel WK, Vuchinich RE, eds. Reframing health behavior change with behavioral economics. Mahwah, NJ: Lawrence Erlbaum, 2000.

37 MacKillop J, Amlung MT, Murphy CM, et al. The behavioral economics of health behavior. In: DiClemente R, Salazar LF, Crosby RA, eds. Health behavior theory for public health: principles, foundations, and applications. Burlington, MA: Jones and Bartlett, 2011:131-62.

38 Schwarzer R, Jerusalem M. Generalized self-efficacy scale. In: Weinman J, Wright S, Johnston M, eds. Measures in health psychology: a user's portfolio casual and control beliefs. Windsor: NFER-Nelson, 1995:35-7.

39 Condiotte MM, Lichtenstein E. Self-efficacy and relapse in smoking cessation programs. J Consult Clin Psychol 1981;49:648-58.

40 Supnick JA, Colletti G. Relapse coping and problem solving training following treatment for smoking. Addict Behav 1984:9:401-4.

41 Etter JF, Prokhorov AV, Perneger TV. Gender differences in the psychological determinants of cigarette smoking. Addiction 2002;97:733-43.

42 Gwaltney CJ, Metrik J, Kahler CW, et al. Self-efficacy and smoking cessation: a metaanalysis. Psychol Addict Behav 2009;23:56-66. 
43 Velicer WF, Diclemente CC, Rossi JS, et al. Relapse situations and self-efficacy: an integrative model. Addict Behav 1990;15:271-83.

44 Nelson W, Reyna VF, Fagerlin A, et al. Clinical implications of numeracy: theory and practice. Ann Behav Med 2008;35:261-74.

45 Reyna VF, Nelson WL, Han PK, et al. How numeracy influences risk comprehension and medical decision making. Psychol Bull 2009;135:943-73.
46 Mantwill S, Monestel-Umaña S, Schulz PJ. The relationship between health literacy and health disparities: a systematic review. PLoS One 2015;10:e0145455.

47 Jamal A, Homa DM, O'Connor E, et al. Current cigarette smoking among adults United States, 2005-2014. Morb Mortal Wkly Rep

2015;64:1233-40. 